\title{
Adjuvante und neoadjuvante Therapie mit Imatinib
}

Der Stellenwert einer adjuvanten Therapie gastrointestinaler Stromatumoren (GIST) wurde und wird in drei randomisierten Studien untersucht. Dabei zeigte die doppelblinde, randomisierte, placebokontrollierte Phase-III-Studie ACOSOG Z9001 eine deutliche Verbesserung des rezidivfreien Überlebens bei Behandlung mit Imatinib ( $p<0,0001$; De Matteo R et al., 2009, Lancet 373: 1097-1104).

Seit Mai 2009 ist Imatinib (Glivec ${ }^{\circledast}$ ) in der Europäischen Union für die Behandlung erwachsener GIST-Patienten mit intermediärem oder hohem Rezidivrisiko zugelassen, deren Tumor vollständig operativ entfernt wurde. Deshalb sollte neben der morphologischen und immunhistochemischen Untersuchung des Tumorgewebes bei allen primären GIST eine Risikoklassifizierung erfolgen. Als Parameter dienen der maximale Tumordurchmesser sowie die Anzahl der mitotischen Zellen in 50 HPFs (High Power Fields). Die primären GIST können in vier Risikoklassen eingeteilt werden: sehr niedriges, niedriges, intermediäres und hohes Rezidivrisiko.

Wie die Daten von Miettinen und Lasota (Miettinen M, Lasota J, 2006, Sem Diagn Pathol 23: 91-102) zeigten, ist auch die Lokalisation des Tumors als drittes Kriterium prognostisch relevant. So weisen nicht gastrische GIST im Vergleich zu primär im Magen lokalisierten Tumoren ein aggressiveres klinisches Verhalten auf.
Für einen Tumor im Rektum ergibt sich nach der Klassifikation von Miettinen und Lasota ein hohes Rezidivrisiko. Da es sich um eine retrospektive Studie in einem einzelnen Zentrum handelt, müssen diese Ergebnisse durch weitere Studien validiert werden.

\begin{abstract}
Neoadjuvante Imatinib-Therapie
Bei zunächst nicht resezierbaren oder nur durch multiviszerale Resektion operablen Tumoren kann Imatinib neoadjuvant (präoperativ) eingesetzt werden. Aufgrund des häufig verzögerten bildgebend nachweisbaren Ansprechens sind als minimale Behandlungsdauer mindestens 4 Monate bis 6 Monate sinnvoll. Ziel dieser Behandlung ist es, nach dem maximalen Therapieansprechen funktions- und organerhaltend operieren zu können. SH
\end{abstract}

Quelle: Informationen der Novartis Pharma GmbH

\section{ErbB2-positives Mammakarzinom}

\section{VITAL-Studie: Lapatinib plus Vinorelbin}

\begin{abstract}
Der Tyrosinkinase-Inhibitor Lapatinib wurde Mitte 2008 zur Therapie bei ErbB2 (HER2= Human Epidermal Growth Factor Receptor 2)-überexprimierendem fortgeschrittenem oder metastasiertem Mammakarzinom zugelassen. Basis der Zulassung war die Phase-III-Studie EGF100151 (Geyer CE et al., 2006, N Engl J Med 355: 2733-2743; Cameron D et al., 2008, Breast Can Res Treat 112: 533-543): In dieser Studie erwies sich Lapatinib/ Capecitabin bei MammakarzinomPatientinnen, die bereits eine Vortherapie mit Anthrazyklinen, Taxanen und Trastuzumab erhalten hatten, der Capecitabin-Monotherapie beim primären Endpunkt Zeit bis zur Progression als überlegen.
\end{abstract}

Jetzt wird eine neue Kombination mit Lapatinib $\left(\right.$ Tyverb $^{\oplus}$ ) geprüft. VITAL (LAP112620) ist eine Phase-II-Studie, für die 105 vorbehandelte Patientinnen mit ErbB2-positivem, metastasiertem Mammakarzinom rekrutiert und randomisiert im Verhältnis 1:2 mit Lapa-
tinib/Capecitabin oder mit Lapatinib/Vinorelbin behandelt werden sollen (Abb.).

Lapatinib/Vinorelbin wird bereits in zwei Dosisfindungsstudien geprüft. Erste Ergebnisse zeigen, dass die kontinuierliche Gabe von Lapatinib in einer Dosis von $1.250 \mathrm{mg}$ täglich mit 20mg/m区 Vinorelbin an Tag 1 und 8 in dreiwöchigen Zyklen sicher und verträglich ist. Die Verträglichkeit dieser Kombination wird in einer initialen Sicherheitsphase der VITALStudie weiter überprüft.

In Deutschland sind zehn Prüfzentren an der VITAL-Studie beteiligt; die Rekrutierung wird voraussichtlich bis zum Spätsommer/Herbst 2010 andauern. Eingeschlossen werden können Patientinnen mit ErbB2-überexprimierendem, metastasiertem Mammakarzinom, bei denen es nach einer Therapie mit Anthrazyklinen, Taxanen und Trastuzumab zur Progression gekommen ist. Nicht palliativ vorbehandelte Patientinnen können rekrutiert werden, wenn sie Trastuzumab bereits in der Adjuvans erhielten oder wenn Tras-

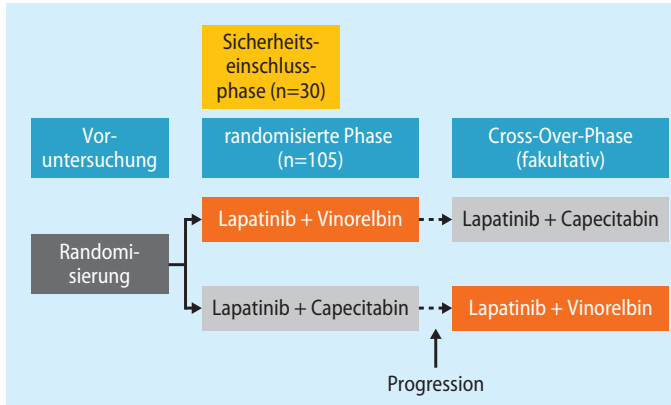

modifiziert nach Informationen der GlaxoSmithKline GmbH \& Co. KG

Studiendesign der VITAL-Studie (LAP112620)

tuzumab kontraindiziert ist. Der positive ErbB2-Status muss immunhistochemisch oder durch Fluoreszenz-in-situ-Hybridisierung (FISH) bzw. chromogener In-situ-Hybridisierung (CISH) gesichert sein.

Informationen zur Studie und den beteiligten Zentren sind telefonisch unter 0180/3456100 (9 Cent/Min., täglich von 9.00-17.00 Uhr) sowie unter www.brustkrebs-studien.com erhältlich. $K A$

Quelle: Informationen der GlaxoSmithKline GmbH \& Co. KG 